\title{
Algebraic Specification and Development in Geometric Modeling*
}

\author{
Y. Bertrand, J.-F. Dufourd, J. Françon, P. Lienhardt
}

\begin{abstract}
Département d'Informatique, Université Louis-Pasteur / CNRS
7, rue René-Descartes, 67084 Strasbourg Cedex, France, tel: 33886143 00, e-mail: \{bertrand, dufourd, françon, lienhardt\} @dpt-info.u-strasbg.fr
\end{abstract}

\begin{abstract}
For several years now, the Geometric Modeling Group of Strasbourg has been working on new formal concepts and tools for describing and manipulating the boundary representation of geometric objects. In a large project of an interactive modeller for volumic objects, the description of which is based on generalized maps, it attempts to cover the whole process from mathematical modeling to efficient implementation, via a complete algebraic specification. Basic concepts and results of this experiment in horizontal and vertical software specification and development are presented along with several illustrations. Advances in algebraic specification methodology are highlighted, specially hierarchical construction of ordered sorts and operations.
\end{abstract}

\section{Introduction}

For geometrical applications, algebraic specifications improve modeling and software development processes. We try to show it through the report of a large-size project.

Several methods have been proposed for describing and manipulating geometric objects on computers [41, 42]. One of them, namely boundary representation $[2,38,39]$, consists in describing objects by their subdivisions, in vertices, edges, faces and volumes. In this frame, mathematical combinatorial models have been proposed. The oldest one is the combinatorial map [16], well studied in [28, 44, 43, 8]. It has numerous uses in geometric modeling $[1,31,21,11]$. New combinatorial topological models of $n$ dimensional objects rely on map extensions $[9,29,32,13]$. Among them, we have proposed the n-dimensional generalized map, or n-g-map $[33,34,35]$. It is a particularly efficient model to deal with topology of manifolds. When completed by an embedding model, it allows to describe the entire geometry of n-dimensional manifold objects.

Furthermore, for a long time, it has been attempted to improve the computer graphics programming techniques by functional [40], logical [20], or object-oriented [26] approaches. But we think that decisive progress will come above all from formal specification techniques, particularly algebraic ones $[17,3,46]$. Their first outstanding use was in [36, 37] where graphical basic objects and operations were algebraically specified. Other works normalize libraries of graphical interactive primitives, like GKS [10], or describe particular algorithms [30]. Note also the attempt of [25] which specifies elementary geometric constructions with an extended OBJ3. In $[12,13,14]$, we give the basis of an algebraic specification of maps and extensions. However using algebraic specifications has never been reported for computer graphics real-size applications.

So, for a full-scale test of both the operationality of the n-g-map model and the efficiency of the algebraic specification techniques, our group has designed and developed original and complex software, namely an interactive volumic modeller, i.e., a program which helps to interactively build and handle 3D geometrical objects, with topological basis [5]. The algebraic specification of this software is the continuation of our preceeding work. It gives us a rigorous hierarchical description of object sorts and operations, with a functional constructive point of view, an horizontal structuring, a vertical development

\footnotetext{
${ }^{*}$ This research has been supported by the GDR Programmation, CNRS, France.
} 
[18], and even a logical prototyping [3] and a real implementation. As far as we know, this is the first attempt to develop an interactive volumic modeller on a topological basis, and, moreover, with the help of a complete algebraic specification. This kind of product is the kernel of specialized software for industrial applications, e.g., mechanical CAD, 3D meshing, design, architecture.

We present here the $n-g$-maps and the modeller. However, the paper is especially centered on the specification problem and on the solutions supplied. We insist on the theoretical fundamentals, the methodology of construction of the ordered sorts and the specification of the main operations. We rapidly discuss a few choices we made on realization, interactivity and implementation. The power of the modeller is illustrated by complex geometric objects.

Section 2 describes the embedded n-g-maps, derived notions and properties. Section 3 presents the main functionalities and user operations of the modeller. Section 4 proposes an order-sorted kernel of algebraic specification for the modeller. Section 5 deals with the vertical development. Section 6 treats the horizontal structuring. Section 7 presents technical features of the modeller. Section 8 summarizes this experiment and gives future prospects. An Appendix shows screen pictures of objects built by the modeller.

\section{A Geometrical Model}

\subsection{A Topological Model}

The $n$-dimensional generalized map, $n$-g-map for short, is a combinatorial notion, which is used as a general basis for the topological modeling in dimension $n$. The mathematical elements we give here are only those used in the following. A complete study as well as justifications for modeling and mathematical proofs of soundness are in $[33,34,35]$.

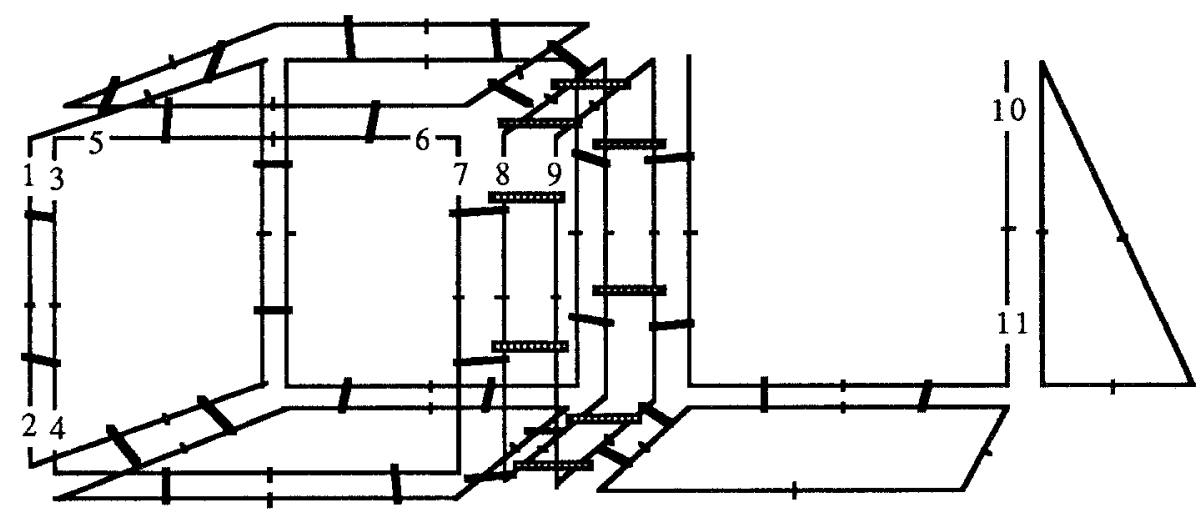

Fig. 1. An example of a 3-g-map with 2 connected components.

Recall that an involution $\alpha$ in a set $\mathrm{D}$ is a bijection such that $\alpha(\alpha(\mathrm{x}))=\mathrm{x}$ for any $\mathrm{x}$ in $\mathrm{D}$. Let $\mathrm{n} \geq-1$. An $n-g-$ map $\mathrm{G}=\left(\mathrm{D}, \alpha_{0}, \ldots, \alpha_{\mathrm{n}}\right)$ consists of a finite set $\mathrm{D}$ of darts with $\mathrm{n}+1$ companion involutions $\alpha_{k}$ in $D, 0 \leq k \leq n$, with the constraints: $\alpha_{k} o \alpha_{j}$ is an involution, for $\mathrm{k}+2 \leq \mathrm{j} \leq \mathrm{n}$. Darts $\mathrm{x}$ and $\mathrm{y}$ are said to be $k$-sewn in $\mathrm{G}$ if $\alpha_{\mathrm{k}}(\mathrm{x})=\mathrm{y}$ (and $\alpha_{\mathrm{k}}(\mathrm{y})=\mathrm{x}$ ).

Fig. 1 (and Picture 1 in Appendix) shows a 3-g-map represented, or embedded, in $\mathbf{R}^{3}$. Darts are natural numbers (only partially written in the drawing for clarity) embedded as half straight line segments in $\mathbf{R}^{3}$. Involution $\alpha_{0}$ is symbolized by thin strokes between half segments, $\alpha_{1}$ by their junctions, $\alpha_{2}$ by thicker black strokes, and $\alpha_{3}$ by hatched strokes. Thus $\alpha_{0}(1)=2, \alpha_{0}(2)=1, \alpha_{1}(3)=5, \alpha_{2}(1)=3, \alpha_{2}(8)=7, \alpha_{3}(8)=9, \alpha_{3}(9)=8$, $\alpha_{1}(10)=10, \alpha_{2}(9)=9, \alpha_{3}(1)=1$, etc. 
An $\mathrm{n}$-g-map can be viewed as a multigraph in $\mathrm{D}$ with $\mathrm{n}+1$ companion symmetric binary relations determined by the $\alpha_{\mathrm{k}}$, for $0 \leq \mathrm{k} \leq \mathrm{n}$. The result is a notion of (strong) connected component for n-g-map as in multigraphs (cf. Fig. 1). The connected component of dart $x$ in $\mathrm{G}$ is denoted $\left\langle\alpha_{0}, \ldots, \alpha_{\mathrm{n}}\right\rangle(\mathrm{x})$. New simple notions can also be introduced.

For $0 \leq \mathrm{k} \leq \mathrm{n}$, the $(n-I)$-g-map of $k$-cells of the $\mathrm{n}-\mathrm{g}$-map $\mathrm{G}=\left(\mathrm{D}, \alpha_{0}, \ldots, \alpha_{\mathrm{n}}\right)$ is defined by $G_{k}=\left(D, \alpha_{0}, \ldots, \alpha_{k-1}, \alpha_{k+1}, \ldots, \alpha_{n}\right)$. The $k$-cell of $\mathbf{G}$ incident to dart $\mathrm{x}$ is the connected component of $\mathrm{x}$ in $\mathrm{G}_{\mathbf{k}}$. The 0-cells, 1-cells, 2-cells and 3-cells of $\mathrm{G}$ are respectively called the vertices, edges, faces and volumes of $\mathrm{G}$ (Fig. 2).

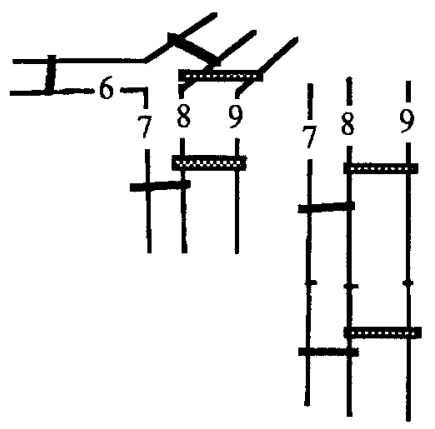

(a) A vertice

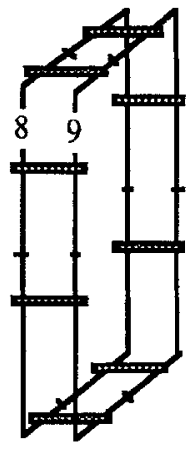

(c) A face

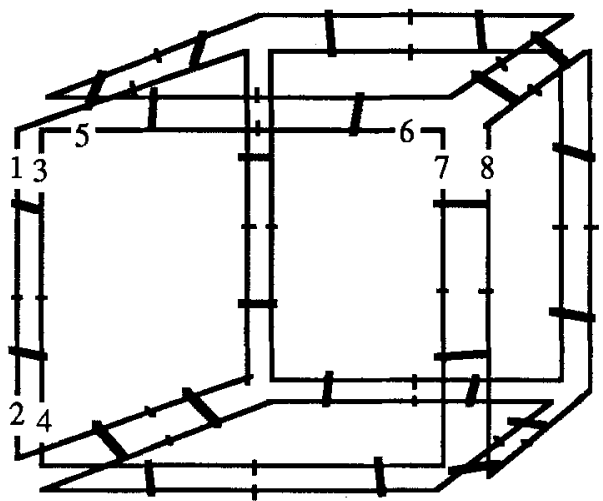

(d) A volume

Fig. 2. K-cells incident to dart 8 of the 3-g-map in Fig. 1.

Similarly, for $0 \leq \mathrm{k} \leq \mathrm{n}$, the (k-1)-g-map of simple $k$-cells of $\mathrm{G}$ is defined by $\mathrm{G}_{\mathrm{k}}^{\prime}=\left(\mathrm{D}, \alpha_{0}\right.$, $\left.\ldots, \alpha_{\mathrm{k}-1}\right)$. The simple $k$-cell of $\mathrm{G}$ incident to dart $\mathrm{x}$ is the connected component of $\mathrm{x}$ in $\mathrm{G}_{\mathrm{k}}$ (Fig. 3 ). So, constraints $\alpha_{k} \circ \alpha_{j}$ is an involution of the $n-g$-map definition say that $k$ sewings are always done in $\mathrm{n}-\mathrm{g}$-maps for all the darts of isomorphic simple k-cells.

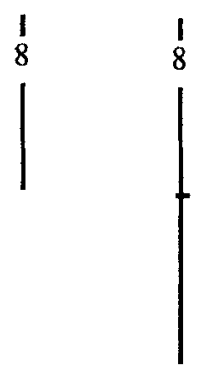

(a) A s. vertex

(b) A s. edge

(c) A s. face

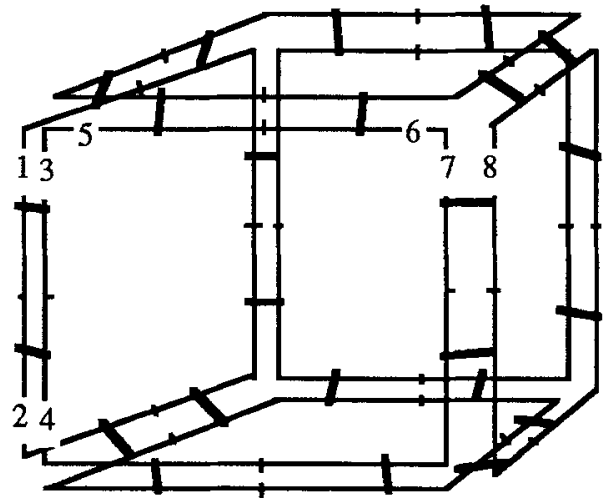

(d) A simple volume (= a volume)

Fig. 3. Simple (or s.) k-cells incident to dart 8 of the 3-g-map in Fig. 1.

\subsection{A Geometrical Embedding Model}

For any dimension $\mathrm{k}, 0 \leq \mathrm{k} \leq \mathrm{n}$, the geometric $k$-embedding of an $\mathrm{n}$-g-map in an $\mathrm{n}$ dimensional Euclidean space $\mathbf{R}^{\mathbf{n}}$ is fixed by labels associated with $\mathbf{k}$-cells, in fact by a single dart per $\mathrm{k}$-cell. 
More precisely, for $0 \leq \mathrm{k} \leq \mathrm{n}, k$-embedding labels are manifolds of dimension $\mathrm{k}$, i.e., in dimensions $0,1,2$, etc, respectively points, Jordan arcs, disc homeomorphic patches, etc. In Fig. 1, each vertex is 0 -embedded in a point of $\mathbf{R}^{3}$, each edge as a straight line segment, each face as a plane patch, and each volume as a polyhedron. Note that the display often splits the cells, to show darts and sewings, as in Fig. 1 and Picture 1.

\subsection{Properties of the Generalized Maps}

Many interesting properties about the topology of objects can be obtained from a modeling by n-g-maps. The principal ones concern 2 -g-maps which modelize surfaces. Indeed, with a given 2-g-map may always be associated the topology of a subdivision of a surface, open or closed, orientable or not, with or without boundaries. Conversely, a 2-gmap may always be associated with the topology of any surface subdivision $[27,33]$.

Three characteristics of a 2-g-map are typical of the subdivision of the surface whose topology is represented by the 2-g-map. They are the number of boundaries, the orientability factor (the value of which is 0 if the surface is orientable, 1 or 2 otherwise), the genus (i.e., the number of holes) [27]. For instance, the triplet of characteristics of a disk, a sphere, a torus, a Moëbius strip, a Klein bottle, respectively are $(1,0,0),(0,0,0)$, $(0,0,1),(1,1,0),(0,2,0)$. These characteristics can be computed from a 2-g-map associated with the surface. Picture 2 shows non orientable open and closed surfaces.

\section{Functionalities of the Modeller}

The modeller is a program which allows interactively to construct, modify, manipulate, display and record geometric 3D objects, thanks to numerous original facilities.

\subsection{Modeled Objects}

The end user of the modeller manipulates embedded 3-g-maps with some additional constraints. They modelize objects composed of vertices, edges, faces and volumes, which are regularized or not [45]. Thus, dangling edges, open faces or volumes, non orientable faces can intentionally be manipulated, efficiently detected and possibly discarded.

More precisely, the following additional topological constraints are adopted for the 3g-maps handled by the user: (1) $\alpha_{0}$ is an involution with no fixed point; (2) $\alpha_{0} \circ \alpha_{1}$ has no fixed point; (3) $\alpha_{0} \circ \alpha_{2}$ has no fixed point; (4) $\alpha_{1}(\mathrm{x})=\mathrm{x}$ implies $\alpha_{3}(\mathrm{x})=\mathrm{x}$; (5) $<\alpha_{0}$, $\alpha_{1}>(x)$ and $<\alpha_{0}, \alpha_{1}>\left(\alpha_{3}(x)\right)$ are disjoint for any dart $x$.

The intuitive meaning of these constraints is: (1) dangling darts are forbidden; (2) loops are forbidden; (3) bent edges, i.e., simple edges 2-sewn to themselves, are forbidden; (4) only closed simple faces can be sewn; (5) folded simple faces, i.e., simple faces 3-sewn to themselves, are forbidden. The 3-g-map in Fig. 1 satisfies these properties.

A very simple embedding is adopted: each vertex is embedded as a point of $\mathbf{Q}^{3}$. Thus, an edge is implicitly embedded as the line segment joining the two points associated with the vertices that bound the edge. Similarly, a face is implicitly embedded as a surface patch joining the line segments associated with the edges which bound the face. If the face is bounded by three edges, the patch is planar. Otherwise its exact form is not essential in our application. Such a patch, which is only defined by its boundary, is easily handled by most basic graphics libraries, for instance to fill it or to render it.

\subsection{High-level Operations}

About 150 high-level operations can be used through menus. Creation, deletion, duplication, subdivision, sewing and unsewing of $\mathrm{k}$-cells and connected components of embedded 3-g-maps are among the main supplied facilities. Production and sewing of linearized cubic curves, bicubic surfaces, tricubic volumes, revolution and sweeping curves and surfaces are very useful operations offered for building quickly realistic objects. 
Motion and deformation with constant topology of designated parts of an object are possible under an interactive control. Immediate computing of the three characteristics of built surfaces allows the user to permanently check their quality. Visualization, coloring, picking, files, undo-redo, press book, etc, complete the functionalities.

Despite the simplicity of the topological and embedding models, it is possible to build complex objects with these operations, as displayed in Pictures 3 to 8 of Appendix. In traditional modellers, such objects sometimes need Boolean shape operations [41], which are brute force and time consuming. The clear distinction between topology and embedding in the geometrical model often allows us to avoid these operations. Moreover this distinction is a basic principle of the following formal specifications.

\section{Algebraic Specification of an N-g-map Basic Kernel}

We formalize a kernel of n-g-maps manipulations. It is more general than what is strictly needed for the modeller in order to make specification, subsequent programmation and future re-using more easy, clear and safe. In this section, the specification is mainly an horizontal structuring by extensions without parameter, and without explicitly importinglexporting modules [18]. The specification is order-sorted [23] and equational, with the usual if_then_else_and == (for comparison) polymorphic functional symbols [17]. We always adopt an initial semantics point of view [17, 23]. Specifications of Booleans, non null natural, natural and rational numbers, i.e., Boolean, nznat, nat and rat sorts, are built-in, with the constraint to be in the initial semantics [18].

\subsection{Topology}

The dart sort is named dart. An erroneous dart err dart is introduced with the dart? sort as such that dart < dart?, in the sense of the ordered sorts [23]. We choose atomic n-g-map generators to facilitate the building of other operations by composition, particularly sewings and unsewings of $\mathrm{k}$-cells. That leads to sorts which are greater than necessary and which are called by the generic name map. The specification of the most general sort, map 0 , begins by that of the topological generators, $v, i$, and $l$ :

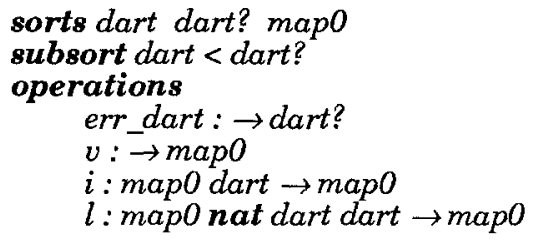

For simplification's sake, we identify here dart sort and nznat, err dart and 0 , and dart? and nat. This enables us to stay in the initial semantics avoiding both parameters and functorial considerations [17]. In fact, darts are actually represented by pointers in the modeller implementation. The symbol of constant $v$ corresponds to the empty map, $i(\mathrm{~m}$, $x$ ) inserts a dart $x$ in a map $m, l(m, k, x, y)$ associates $y$ to $x$ with label (in dimension) $k$, in a "semi-sewing". These operations are total. So, we can insert by $i$ several times the same dart in a map, and apply $l$ anyhow. In initial semantics, the sort map 0 is interpreted as the set of all the closed terms which are generated by these operators.

The following operator $a$ formalizes the n-g-map functions $\alpha_{k}$ (cf. section 2) extended to the maps: $a(m, k, x)$ returns the successor at dimension $k$ of $x$ in the map $m$ :

\section{operation}

$a:$ map0 nat dart $\rightarrow$ dart?

axioms ( $m:$ mapo, $k j:$ nat, $x y z:$ dart)

$a(v, k, z)=$ err dart

$a(i(m, x), k, z)=$ if $z==x$ then $x$ else $a(m, k, z)$

$a(l(m, j, x, y), k, z)=$ if $k==j$ and $z==x$ then $y$ else $a(m, k, z)$ 
To retrieve the usual cases, we define a new mapl sort, with mapl<map 0 , interpreted as a set of directed multigraphs on darts, with edges labelled in nat. The invariant inv_mapl characterizes the maps of map 0 which belong to mapl. The operation $i(m, x)$ is interpreted as the insertion of a graph node $x$ in $m$, and $l(m, k, x, y)$ as the addition of an edge $(x, y)$ labelled by $k$. The Boolean operation $e x$ dart $(m, x)$ returns true iff $x$ is in $m$ :

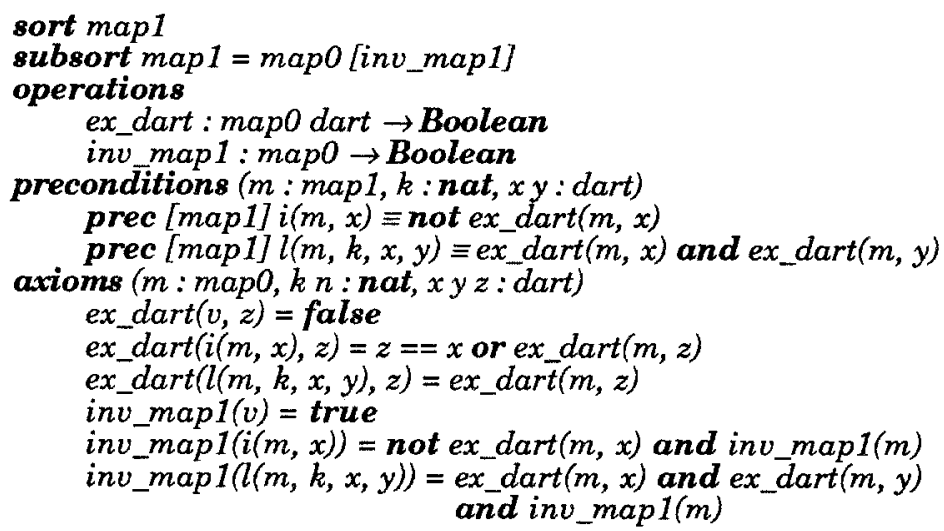

Preconditions for $i$ and $l$ derive from the invariant inv map1: when applied to mapl $m$, $i(m, x)$ returns a legal element of mapl iff $x$ does not belong to $m$, and $l(m, k, x, y)$ iff $x$ and $y$ belong to $m$. Each precondition definition is indexed by the target sort of the operator between square brackets, e.g., [mapl] for $i$. In fact, the above preconditions are the weakest ones to satisfy the invariant, which can be proven. The new operations $i$ and $l$ are restrictions of the old ones: they have exactly the same semantics as previously on the domain defined by the invariant. This satisfies the monotonicity condition of [23].

A hierarchy of about 40 ordered map sorts with invariants and operations with preconditions has been defined. Constraints get more and more restrictive: sorts with $\alpha_{k}$ injective, with $\alpha_{k}$ a permutation, with $\alpha_{k}$ an involution, with $k \leq 3$, with embedding constraints, etc. For instance, the sort map3 take into account the permutativity of $i$ and $l$ :

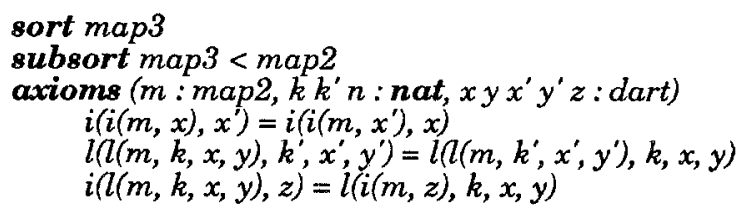

The sort hierarchy meets the order-sorted signature constraints: it is regular and coherent, in the sense of [23]. It is completely described in [4].

\subsection{Embedding}

We extend the specification with an embedding generator em. Thus, em( $m, k, x, q)$ embeds the $k$-cell of $m$ containing dart $x$ on a geometric object $q$ of sort embed and of dimension $k$ :

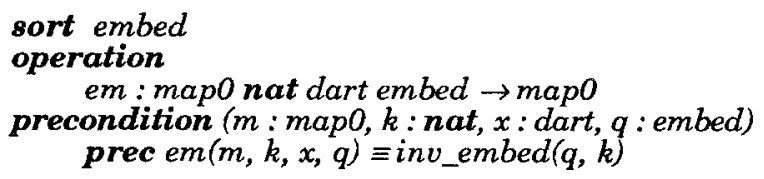

To meet this constraint, we introduce sorts embedk of geometric objects of dimension $k$, with embedk<embed. We also define an invariant inv_embed $(q, k)$, true iff $q$ belongs to sort embedk, which is the precondition for $\mathrm{em}$. In fact, only 0 -embeddings are explicit 
in the modeller. They are 3D points, i.e., triplets of rat coordinates, built by the 0 embedding generator genembed 0 . Thus we have in this simple case:

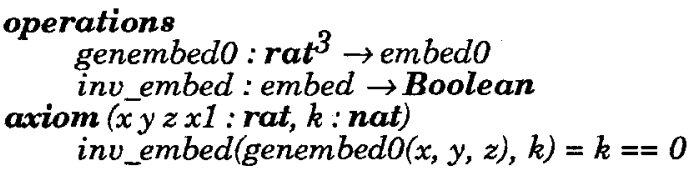

The introduction of the map generator em enriches the set of map 0 terms. It also forces to complete the previous specifications. For instance, a new axiom is needed for $a$ :

axiom (m:mapo, $k j:$ nat, $x z:$ dart, $q:$ embed) $a(e m(m, k, x, q), j, z)=a(m, j, z)$

Such axioms can always be automatically added, and wont be written here anymore. In fact, as there are thousands in the modeller specification, it is impossible to write them by hand. They are called implicit axioms in [37]. As previously, only darts present in a map can be embedded. This property restricts the map0 sort to a new sort mapel < map0 Furthermore, for the modeller, at most one dart per $k$-cell is $k$-embedded. Thus, we introduce again a new sort mape $2<$ mape1, defined by an invariant inv_mape2, in fact free_embed $(m, k, x)$, that is true iff no dart of the $k$-cell of $x$ is $k$-embedded in $m$ :

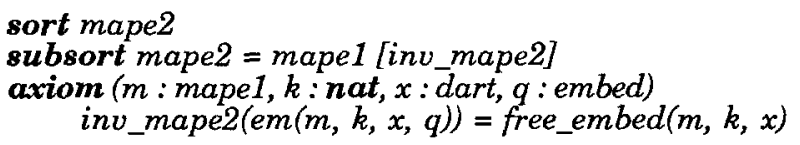

We immediately deduce from this invariant the weakest precondition of operator $\mathrm{em}$ for the mape 2 sort. At this level, the precondition for $l$ is more tricky to obtain: $l(m, k, x, y)$ is legal w.r.t. the embedding constraints only if, for all $j \neq k$, the $j$-cells of $x$ and $y$ are still the same ones, or one of them is not $j$-embedded. The auxiliary operation free_embedl $(m$, $k, x, y)$ returns true iff this condition holds. Its formal specification is not given here.

\subsection{Geometric Operators}

We impose to the final objects of the modeller exactly one k-embedding per $\mathrm{k}$-cell. A new sort mape $3<$ mape 2 derives. To maintain the mape 3 invariant, operator $l$ might be called by a new operator $l l$ which removes some embeddings to avoid two distinct $\mathrm{k}$-embeddings for a k-cell. Thus, operation $l l(m, k, x, y)$ removes, if necessary, the embeddings of the cells which contains $y$, and $k$-sews $x$ and $y$ by $l(l(m, k, x, y), k, y, x)$. Precisely, before the $k$-sewing, for all $j \neq k$, it calls a function $\operatorname{llk}(m, j, x, y)$ which removes if necessary the $j$ embedding associated with the $j$-cell containing $y$.

Symmetrically, function $r r(m, k, x)$ first unsews in $m$ darts $x$ and $y=a(m, k, x)$, then duplicates if necessary the $j$-embeddings for all $j \neq k$ if $x$ is $j$-embedded in $m$. It calls for all $j$ $\neq k$ a function $r r k(m, k, j, x, y)$ which duplicates the $j$-embedding if necessary.

\subsection{Sort Hierarchy and Initial Semantics}

This leads us to a rather complex map sort hierarchy, with the top level map0. Several branches go down: one for topology, with ... map $2<\operatorname{map} 1<\operatorname{map} 0$, one for embedding, with ...< mape2 < mape1 < map0, and other ones for cell markers (cf. Section 5). Note that new map generators are always introduced at top level, with automatical repercussions on the lower levels by implicit axioms. The 3-g-map sort of the embedded 3-g-maps with the modeller constraints of Section 3 is at the bottom of the hierarchy. In an initial semantics, it can be interpreted as the intersection of all its upper sorts, in other words, as the set of the map 0 terms which exactly satisfy the invariants of the whole hierarchy [4]. 
With order-sorted algebras [23] it is easy to describe step by step numerous geometric models, starting from a unique model with atomic generators. Invariants and preconditions help to easily limit ordered geometric sorts, as pointed out by [25]. Intermediate models correspond to intermediate states when building geometric objects [13]. They facilitate defining total operations. They can also be used in modeling as such, when extending the class of handled objects beyond the strict 3-g-maps [4].

However, our specifications might be considered as over-specified [6]. It is the case with any specification where generators are chosen, sometimes rather early for practical reasons, because some implementations and algorithms are favoured. In fact, an early decision about the choice of the generators allows us to properly define the sort hierarchy and to make the kernel runable, which is essential for debugging.

Moreover, our aim is that the last level of specification be isomorphic with the implementation. That is an additional reason why, contrary to other approaches, as for instance loose semantics [46], we have favoured an initial semantics approach during the whole development of the specifications.

The properties of the 3-g-maps, for instance $\alpha_{k}$ being an involution, for $0 \leq \mathrm{k} \leq 3, \alpha_{0}$ being an involution with no fixed point, $\alpha_{0} \circ \alpha_{1}$ having no fixed point, etc, can be obtained from the specification as inductive theorems. That agrees with the initial semantics. These theorems can be proven by a mechanized inductive reasoning $[14,15]$.

\section{Vertical Development}

The previous operators are combined to obtain new ones. Their definitions can be given first at a high level of abstraction, appropriate for logical prototyping, but often inefficient for a realistic implementation. The new operators have to be refined in a progressive vertical development. Our aim is to keep the same specification framework for this refinement. We examine this process through an example.

We specify operator $c c$ of cell traversal: $c c(m, x)$ extracts the connected component of $m$ of sort mapl containing dart $x$. We start with a short abstract definition à la Kruskal using other operators, the specification of which is simple and only given in comments:

operation

cc : map1 dart $\rightarrow$ map1

precondition $(m: \operatorname{map} 1, x:$ dart $)$

$\operatorname{prec} c c(m, x) \equiv \operatorname{ex} \operatorname{dart}(m, x)$

axioms ( $m: \operatorname{map} 1, k:$ nat, $x y z:$ dart $)$

$c c(i(m, x), z)=$ if $x==z$ then $i(v, z)$ else $c c(m, z)$

$\operatorname{cc}(l(m, k, x, y), z)=$ if islink $(l(m, k, x, y), z, x)$

then if islink $(m, x, y)$ then $l(c c(m, z), k, x, y)$

else l(union $(c c(m, x), c c(m, y)), k, x, y)$

I* union (m1, m2) merges $m 1$ et $m 2$;

else $c c(m, z)$

islink $(m, x, y)$ is true iff the connected components of $x$ and $y$ in $m$ are the same ones *

A straight implementation of $c c$ as specified (a logical prototyping) will always have a time complexity exponential w.r.t. $p$, the number of darts in the map (in the best, average and worst cases). In fact, an acceptable response time imposes a complexity of $c c$ in $O(p)$ in the worst case.

We improve this situation by a refinement of the specification. Thanks to a new mapo generator of dart marking, named mark, we specify a depth-first traversal $m k c c(m, x)$, which marks the connected component of $x$ in $m$ instead of extracting it. The complexity in maximum time of this new operator is in $O(p)$, when supposing an $O(I)$ complexity for the implemented basic operators, which is true in the actual implementation of the modeller. To simplify, we directly pass to a new sort of marked maps, named mapc, such that mapc < mapl. The objects of this sort meet the following constraint of strict connectivity: existence of a path from a dart $x$ to a dart $y$ implies existence of another one from $y$ to $x$. This property also holds for $\mathrm{n}-\mathrm{g}$-maps, so 3-g-map < mapc < mapl: 


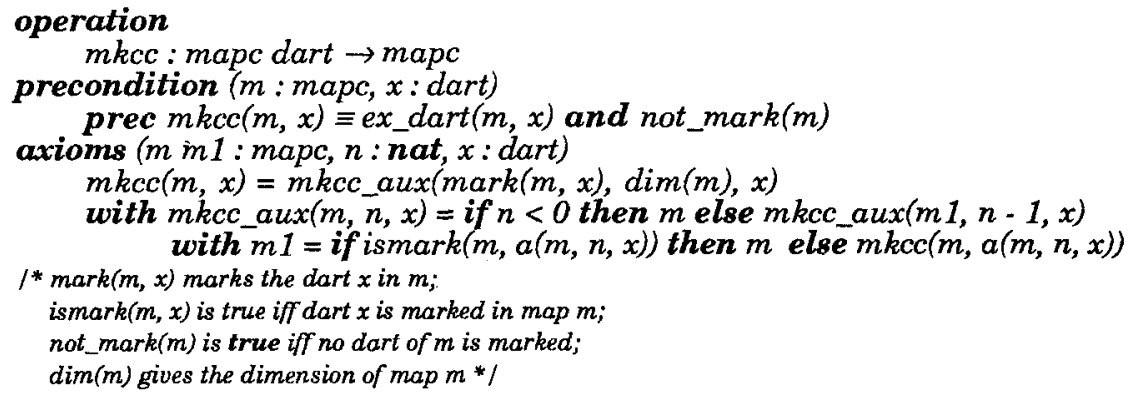

For a final non recursive implementation, we refine once more the specification into another one, which is tail recursive, and directly implementable in $O(p)$ time. Actually, it is easier to realize this traversal in a breadth-first way, the queue of marked darts being explicitly linked in the map itself thanks to another map generator, called markcc:

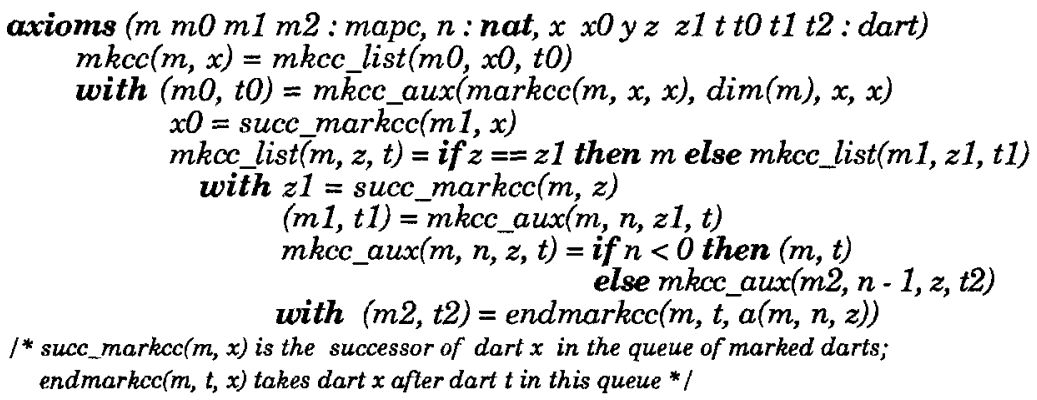

Note that the map0 sort enrichment by new mark generators is only realized with the last version of the generators, i.e., in the example, with markcc. In fact, when we go down the sort hierarchy, for instance fixing $n=3$, or imposing that some involutions be without fixed points, we can write specialized traversal functions still more efficient than the ones above. Thus, an edge traversal in a 3-g-map can be realized by direct compositions of $a$, for dimensions 2 and 3, without explicit depth- or breadth-first traversal. Finally, through factorizing definitions by with and using iterators (cf. Section 6), the specification can be directly translated into a procedural language.

\section{Horizontal Structuring}

\subsection{Sewing Operators}

At 3-g-map level, we can write functions to create, remove, sew and unsew k-cells. We distinguish between topological operators, with strong topological and embedding preconditions, and geometrical (i.e., topological and embedding) operators of higher level, that only have topological preconditions. Particularly, sewing operators which meet the 3-g-maps constraints of the modeller can be defined this way.

We present three examples: topological sewing, se, geometric sewing, gse, and insertion, gie2, of two simple edges, with only the formal specifications of se and gse:

operations

se, gse: 3-g-map dart dart $\rightarrow$ 3-g-map

preconditions ( $g: 3-g-m a p, x y:$ dart)

prec gse $(g, x, y) \equiv e x \_d a r t(g, x)$ and ex_dart $(g, y)$ and $a(g, 2, x)==x$

and $a(g, 2, y)==y$ and $x \neq a(g, 0, y)$ and $x \neq y$

prec se $(g, x, y) \equiv$ prec (gse $(g, x, y))$ and free_embedl $(g, 2, x, y)$

and free_embedl $(g, 2, a(g, \overline{0}, x), a(g, 0, y))$ 


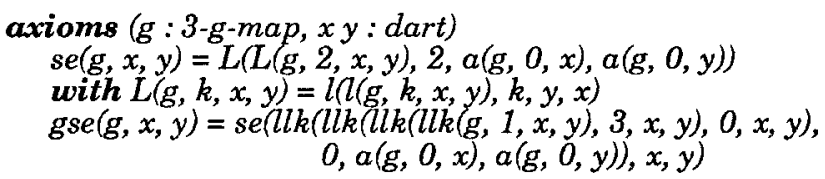

Operator gse is written efficiently by using, instead of the general operator $l l$, operator $l l k$ at the appropriate dimensions to remove superfluous embeddings (cf. subsection 4.3). Operation gie2 $(g, x, y)$, which uses se, is rather surprising. It allows us to subdivide one face or to merge two faces, depending on whether the faces of $x$ and $y$ are the same ones or not (Fig. 4, (a) and (b), and Pictures 9 and 10 in Appendix).

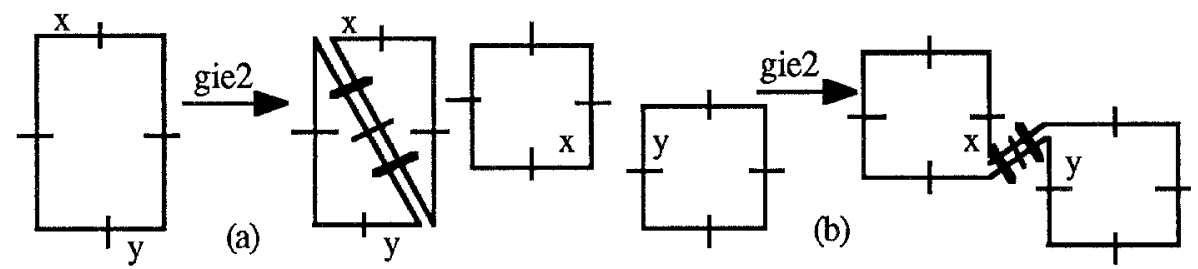

Fig. 4. Insertion of two simple sewn edges.

In the modeller, several useful operators are defined the same way, in particular Euler operators, which keep the surface genus unchanged [39].

\subsection{Meshes, Second Order and Iterators}

We now briefly present the specification of a high-level operator, namely a mesh contructor [4]. The interest lies in the way this operator is specified. It clearly distinguishes between topology and embedding, and reduces the required number of operations. Moreover, it makes possible to justify the introduction of second order functions and iterators in specifications. We propose here to create simple meshes, which can be completed by poles afterwards (Fig. 5, Picture 11, and Picture 12 for an extension).
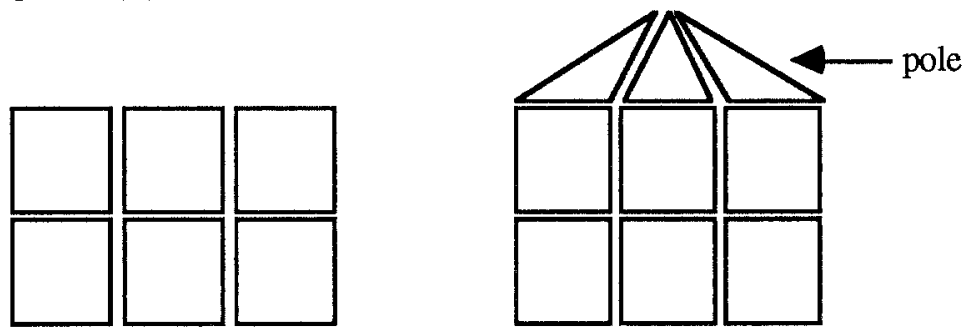

Fig. 5. A simple mesh and a mesh with pole (the 0 and 2-sewings are omitted).

A simple mesh is made of simple quadrangles sewn together by operator se. The mesh topology is progressively created and embedded thanks to a function $f:$ nat $^{4} \rightarrow$ embed which 0 -embeds a dart per quadrangle. The 4 variables of $f$ are $n$ and $p$, which give the mesh size, and $i \in[0, n]$ and $j \in[0, p]$, which give the columns and lines of the mesh points.

The mesh function, denoted grid, uses auxiliary creations of squares, i.e., sqrgrid, sqrgrid11, sqrgridn1 and sqrgridlp, whose specification is omitted (cf. [4] for details):

operation

grid : 3-g-map (nat ${ }^{4} \rightarrow$ embed) nat $^{2} \rightarrow 3-$ g-map

axioms (g:3-g-map, $f:$ nat $^{4} \rightarrow$ embed, $i j k n p:$ nat)

$\operatorname{grid}(g, f, n, p)=\operatorname{grid} 2(\operatorname{grid} 1$ (sqrgrid1 $1(g, f, n, p), f, 2, n, p), f, 2,2, n, p$

with $\operatorname{grid} 1(g, f, k, n, p)=$

if $k>n$ then g else grid1(sqrgridn1(g, $f, k, n, p), f, k+1, n, p$ ) $\operatorname{grid} 2(g, f, i, j, n, p)=$ 
if $i>n$ then if $j \geq p$ then $g$ else grid2(sqrgrid $1 p(g, f, j, n, p), 2, j+1, n, p)$ else grid2(sqrgrid(g, $f, i, j, n, p), i+1, j, n, p)$

Second order functions, like grid, are out of the strict frame of traditional algebraic specifications. They could be avoided by using parameterized modules as in OBJ3 [24]. But it is a heavy solution when the parameters concern only a few functions as it is here the case, where, moreover, the semantics does not create any problems.

The use of auxiliary functions gridl and grid2 to simulate two embedded iterations on the mesh squares by a tail recursion makes the specification heavy and reduces its readability. To remove them, we use iterators, considered as macro-definitions. For instance, $v=v 0$; while not cond $(v) \operatorname{do} \operatorname{succ}(v)$ defines by induction the last value $v$ of a sequence of values, where the initial, current and next ones are respectively $v 0, v$ and $\operatorname{succ}(v)$, and the stop condition $\operatorname{cond}(v)$. It textually replaces the following iter function defined by: $v=i \operatorname{ter}(v 0)$ with iter $(v)=$ if cond $(v)$ then $v$ else iter $(\operatorname{succ}(v))$.

The following specification which redefines grid with two embedded iterators, simplifies the specifications and brings us closer to functional and procedural languages:

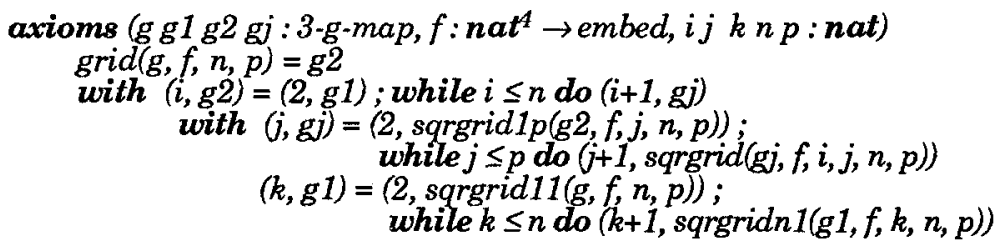

\subsection{Miscellaneous: User Interaction, Errors, Libraries}

Classical algebraic specifications do not conveniently deal with user interactions [37] which can be seen as a distinct process, concurrent with the modeller process. A formalism suited to the description of concurrency, like LOTOS, could be used. In our case, it is a heavy solution, since the entry of external events is strictly limited to a small specification part. We have prefered to describe user interactions by a meta-specification, i.e., a second level of specifications, which manipulate the first level ones by simple axiom modifications [4].

In a modeller, errors are linked to user interaction. Operators with preconditions have been implemented in the way they were specified, i.e., without testing the preconditions in their bodies. They have been encapsulated in high-level functions, algebraically specified too, which deal with computer-human interactions, preconditions and errors.

Today, graphical software development makes an intensive use of built-in graphical libraries. We have formally specified the library we use (Silicon Graphics GL), by an arbitrary choice of basic generators and by expressing other operators w.r.t. them. Such a task was difficult, given the imperfections of the documentation.

\section{Some Technical Features of the Modeller}

The modeller includes about 1800 operations: 1400 for geometric modeling and 400 for the environment and the interface. Most of them, except for 100 mathematical or trivial functions, were developed from complete algebraic specifications. The operations of the basic kernel were translated into PROLOG and tested by logical prototyping [4]. Only 150 parameterized operations are visible for the user through the interface.

The modeller is currently implemented in $\mathrm{C}$ for Silicon Graphics workstations. Thanks to the map concepts, the data structures that are used are very simple. Except for the upper ones, all the map and dart sorts of the hierarchy are really implemented with the same unified $C$ types. A unified map structure consists mainly of a linked linear list of similary dart records, the fields of which are more or less constrained, depending on the invariants 
of the corresponding map sort. Each dart record contains 1 dart pointer to the successor in the list, 4 dart pointers to represent the $k$-sewings, for $0 \leq k \leq 3$, one pointer to the optional 0 -embedding, and several markers for traversal and interactive selections. The program contains about 25000 lines of $\mathrm{C}$, a rather small number for software of this type. This is mainly due to the conciseness and reusability induced by algebraic techniques.

Response time is comparable with those of commercial products with similar features and may be even better. The main operations, particularly the traversals, have a time complexity in $O(p)$, where $p$ is the number of darts. This is not the case in many commercial modellers which often take $O\left(p^{2}\right)$, because they lack directly accessible topological data. Other classical data structures, such as those derived from the "winged edge" [2], often take comparable memory space for a more restricted modeling area.

\section{Conclusion}

The n-g-map is a general and efficient model that helps to describe and handle the topology of n-dimensional objects. With embeddings, this model can be implemented in any dimension. For example, we chose to derive 3-g-maps with 0-embedding for a geometric modeller of complex manifold objects embedded in $\mathbf{Q}^{3}$. Other choices could be made with other map extensions and embeddings. But the handling of non manifold objects [45] requires even more general mathematical concepts, cellular complexes [19] for instance.

With the above algebraic specification and its ordered sorts and invariants, we obtain a formal, functional, hierarchized, and homogeneous description of objects and operations. As often [22], genericity has not been necessary. In the future for instance, it may become interesting, to parameterize the modeller by topological or embedding constraints. Finally, we expect to recover a specification language well suited to the needs above [7].

Proofs of correctness of our horizontal structurings are possible and will be undertaken, with a mechanical help [24]. But, the proofs of correctness of our vertical developments, which involve techniques of program transformation and synthesis, are more difficult to bring into play. Moreover, work about using algebraic specifications in semi-automatical proofs of geometric properties is in progress $[14,15]$.

The concrete results of our study is an interactive modeller of 3D objects. Private firms are already interested by its functionalities. However, it must be adapted and extended in order to be used in real applications. Algebraic specifications give a basis which makes those extentions easier. Among the next fundamental ones, there is the cellular complexes [19] and the Boolean shape operators [41].

Our experiment illustrates how algebraic specifications help to elaborate and study models in geometry, and to design and develop software in this area. Conversely, the spectacular workbench of geometric modeling is convenient to understand how algebraic specifications improve axiomatization and programming processes in a complex field.

\section{References}

1. Baudelaire, $P$ and Gangnet, M: Planar Maps: An Interaction Paradigm for Graphic Design. Proc. CHI'89 (1989) 313-318

2. Baumgart, B: A Polyhedron Representation for Computer Vision. Proc. AFIPS Nat. Conf. Proc. 44 (1975) 589-596

3. Bergstra, J A, Heering, J and Klint, P: Algebraic Specification. ACM, Addison-Wesley (1988)

4. Bertrand, Y: Spécification Algébrique et Réalisation d'un Modeleur Interactif de Subdivisions Tridimensionnelles. Thèse de Doctorat, CRI-ULP, Strasbourg (1992)

5. Bertrand, Y, Dufourd, J-F, Françon, J et Lienhardt P: Modélisation Volumique à Base Topologique. Proc. Micad, Paris (1992)

6. Bidoit, M: Development of Modular Specification by Stepwise Refinements using the PLUSS Specification Language. Tech. rep. LIENS 91-9 (1991),

7. Bidoit, M, Kreowski, H-J, Lescanne, P, Orejas, F and Sannella, D: Algebraic System Specification and Development. LNCS n'501, Springer-Verlag (1991) 
8. Bryant, $R$ and Singerman, D: Foundations of the Theory of Maps on Surfaces with Boundaries. Quart. Journal of Math. Oxford Vol $2 \mathrm{n}^{\circ} 36$ (1985) 17-41

9. Cori, R: Un code pour les graphes planaires et ses applications. Astérisque $\mathrm{n}^{\circ} 27(1975)$

10. Duce, D A , Fielding, E V C and Marshall, L S: Formal Specification of a Small Example based on GKS. ACM Trans. on Graphics Vol 7 n $^{\circ} 3$ (1988) 180-197

11. Dufourd, J-F, Gross, C and Spehner, J-C: A Digitisation Algorithm for the Entry of Planar Maps. Proc. Comp. Graphics Int. Leeds, Springer-Verlag (1989) 649-662

12. Dufourd, J-F: Algebraic Map-Based Topological Kemel for Polyhedron Modelers. Proc. Eurographics, Hamburg, Elsevier (1989) 301-312

13. Dufourd, J-F: Formal Specification of Subdivisions using Hypermaps. Computer Aided Design, Butterworth-Heinemann Vol $23 n^{\circ} 2$ (1991) 99-116

14: Dufourd, J-F: An OBJ3 Functional Specification for the Boundary Representation. Proc. ACMSiggraph Symp. on Solid Modeling Foundations \& CAD/CAM Appl., Austin (1991) $61-72$

15. Dufourd, J-F: Foundations of Boundary Representation Revisited with a New Foremap Axiomatics. Proc. Eurographics Work. on Formal Spec. in Comp. Graphics, Marina di Carrara (1991)

16. Edmonds, J: A Combinatorial Representation for Polyhedral Surfaces. Not. AMS Vol 7 (1960)

17. Ehrig, H and Mahr, B: Fundamentals of Algebraic Specifications Vol 1 : Equations and Initial Semantics. Springer-Verlag (1985)

18. Ehrig, H and Mahr, B: Fundamentals of Algebraic Specifications Vol 2 : Module Specifications and Constraints. Springer-Verlag (1990)

19. Elter, H et Lienhardt, P: Extension de la Notion de Carte pour la Représentation de la Topologie d'Objets Géométriques Complexes. Proc. Journées Gros-Plan, Lille (1991)

20. Franklin, W R, Wu ,P Y F \& Samaddar, S: Prolog \& Geometry Projects. IEEE CG \& A Vol 6 (1986)

21. Gangnet, M, Hervé, J-C, Pudet, T and Van Thong, J-M: Incremental Computation of Planar Maps. ACM Computer Graphics Vol $23 \mathrm{n}^{\circ} 3$ (1989) 345-354

22. Gaudel, M-C: Structuring and Modularizing Algebraic Specifications. R. 01-92 LRI, Orsay (1992)

23. Goguen, J A \& Meseguer, J: Order-Sorted Algebra I: Equational Deduction for Multiple Inheritance, Overloading, Exceptions, and Partial Operations. Tech. Rep.n ${ }^{\circ}$ 89-10 SRI-CSL (1989)

24. Goguen, J A and Winkler, T: Introducing OBJ3. Tech. Rep. $n^{\circ} 88-9$, SRI-CSL Menlo Park (1988)

25. Goguen, J A: Modular Algebraic Specification of Some Basic Geometrical Constructions. Artificial Intelligence Vol 37 (1988) 123-153

26. Grant, E, Amburn, $\mathrm{P}$ and Whitted, T: Exploiting Classes in Modeling and Display Software. IEEE $C G \& A$ Vol 6 (1986) 13-20

27. Griffiths, H-B: Surfaces, Cambridge Univ. Press, Cambridge (1981)

28. Jacques, A: Constellations et Graphes Topologiques. Coll. Math. Soc. J. Bolyai (1970) 657-672

29. James, L: Maps and Hypermaps: Operations and Symmetry. PhD thesis, Dep. of Mathematics, Univ. of Southampton (1985)

30. Lakshminarasimhan and A L, Srivas, M: A Framework for Functional Specification and Transformation of Hidden Surface Elimination Algorithms. CG. Forum Vol $8 n^{\circ} 2$ (1989) 75-98

31. Lienhardt, P: Free-Form Surfaces Modeling by Evolution Simulation. Proc. Eurographics, Nice, Elsevier (1988) 327-341

32. Lienhardt, P: Extension of the Notion of Map and Subdivision of Three Dimensional Space. Proc. STACS, Bordeaux, LNCS Vol 294, Springer-Verlag (1988) 301-311

33. Lienhardt, P : Subdivisions of Surfaces and Generalized Maps. Proc. Eurographics, Hamburg, Elsevier (1989) 439-452

34. Lienhardt, P: Subdivisions of N-Dimensional Spaces and N-Dimensional Generalized Maps. Proc. $5^{\circ}$ ACM Symp. on Comp. Geometry, Saarbrücken (1989) 228-236

35. Lienhardt, P: Topological Models for Boundary Representation : A Comparison with $N$ dimensional Generalized Maps. Comp.-Aided Design Vol 23 n $^{\circ} 1$, Butterworth-H. (1991) 59-82

36. Mallgren, W R: Formal Specification of Graphic Data Types. ACM TOPLAS Vol $4 \mathrm{n}^{\circ} 4$ (1982) 687710 .

37. Mallgren, W R: Formal Specification of Interactive Graphics Programming Languages. ACM Distinguished Dissertation, MTT Press (1982)

38. Mäntylä, $\mathrm{M}$ and Sulonen, R: GWB : A Solid Modeler with Euler Operators. IEEE CG \& A Vol $2 \mathrm{n}^{\circ}$ 7 (1982) 17-31

39. Mäntylä, M: An Introduction to Solid Modeling. Computer Science Press, Rockville (1988)

40. Parsons, M S: Image Representations Using Miranda Laws. Comp. Graphics Forum Vol 8 n $^{\circ} 2$, North-Holland (1989) 99-106

41. Requicha, A: Representations for Rigid Solids : Theory, Methods and Systems. ACM Computing Surveys Vol $12 \mathrm{n}^{\circ} 4$ (1980) 437-464

42. Requicha, A A G and Voelker, H B: Solid Modeling: Current Status and Research Directions. IEEE $C G \& A$ Vol 3 n $^{\circ} 7$ (1983) 25-37

43. Tutte, W: Graph Theory. Encyclop. of Mathematics and its Applications, Addison-Wesley (1984)

44. Vince, A: Combinatorial Maps. J. of Combinatorial Theory Series B $n^{\circ} 34$ (1983) 1-21

45. Weiler, K: The Radial Edge Structure: A Topological Representation for Non-Manifold Geometric Boundary Modeling. in Geometric Modeling for CAD Applications, Elsevier (1988) 3-36

46. Wirsing, M: Algebraic Specification. Handbook of Theoretical Computer Science, Vol 2 : Formal Models and Semantics, Elsevier (1990) 675-788 


\section{Appendix}
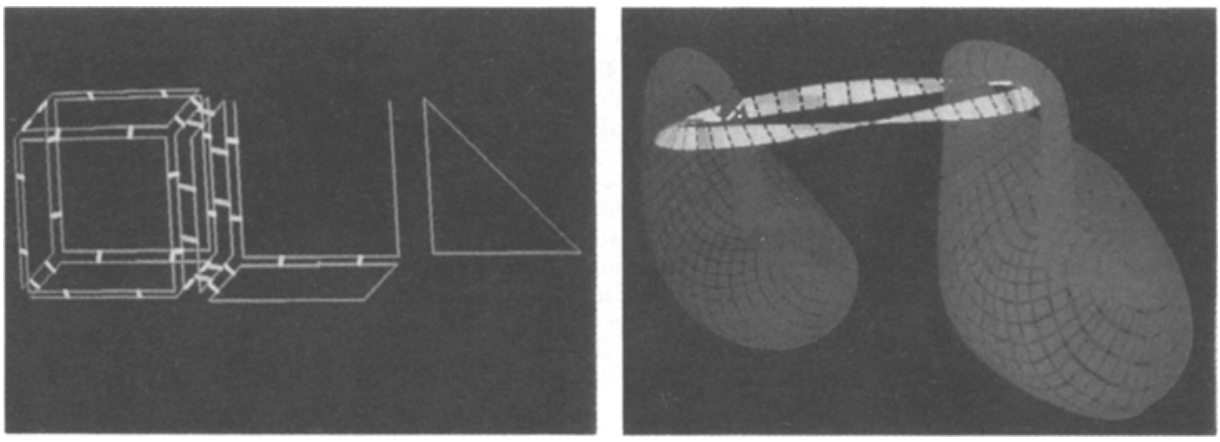

Picture 1: The 3-g-map of Figure 1

Picture 2: A Moz̈bius band and 2 Klein bottles

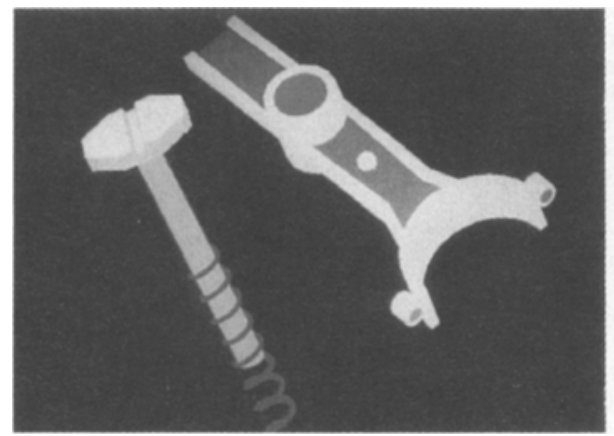

Picture 3: Mechanical parts

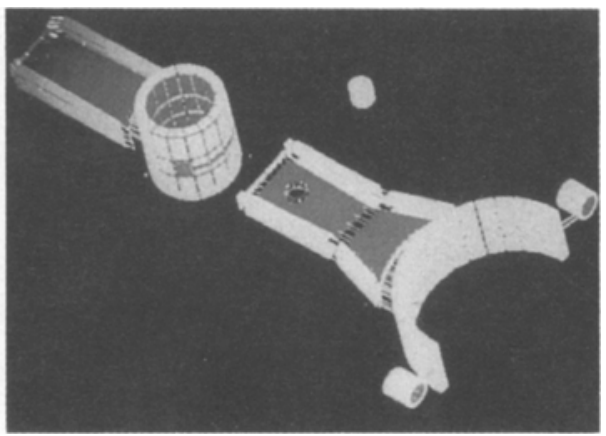

Picture 4: Details of a splitted mechanical part

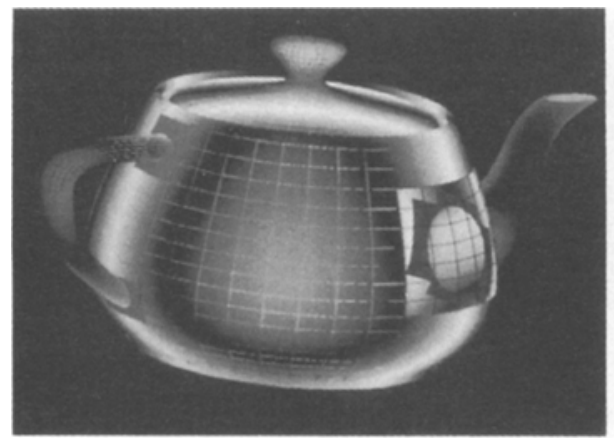

Picture 5: A surfacic teapot

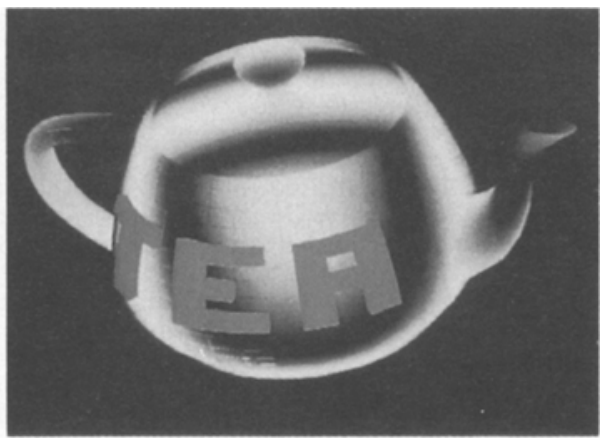

Picture 6: A volumic teapot 


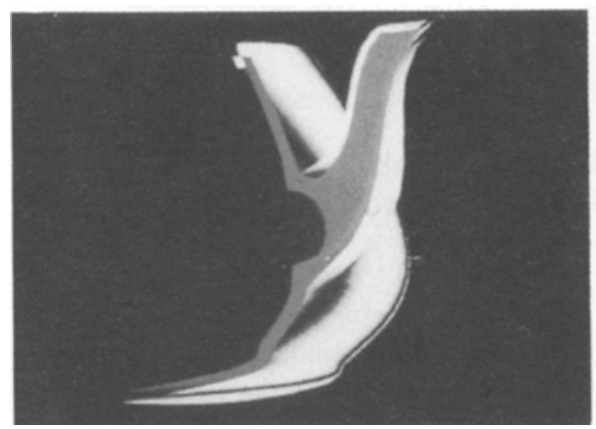

Picture 7: Spout of the volumic teapot

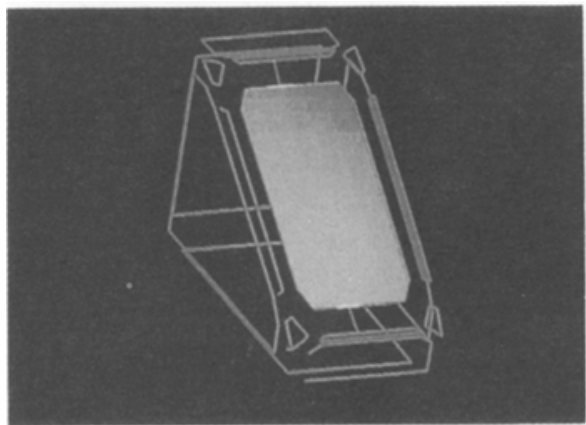

Picture 9: A face to be split

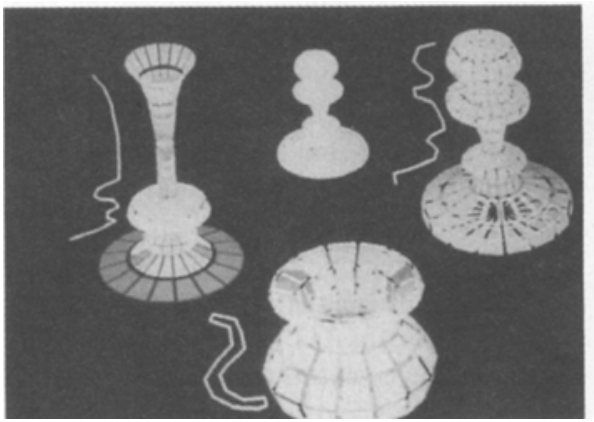

Picture 11: Surfacic meshes

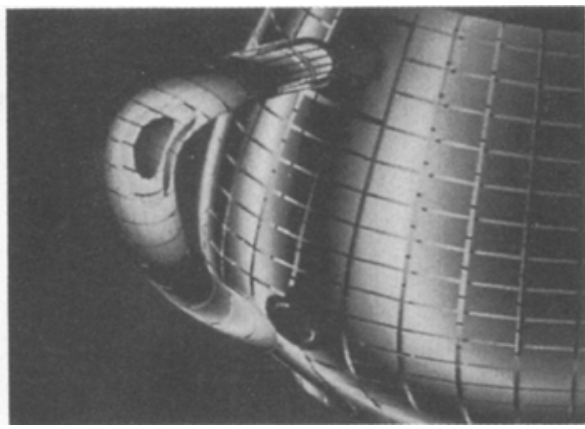

Picture 8: Handle of the volumic teapot

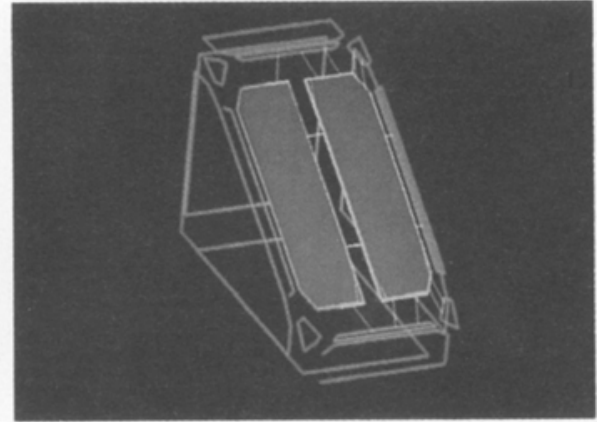

Picture 10: The splitted face

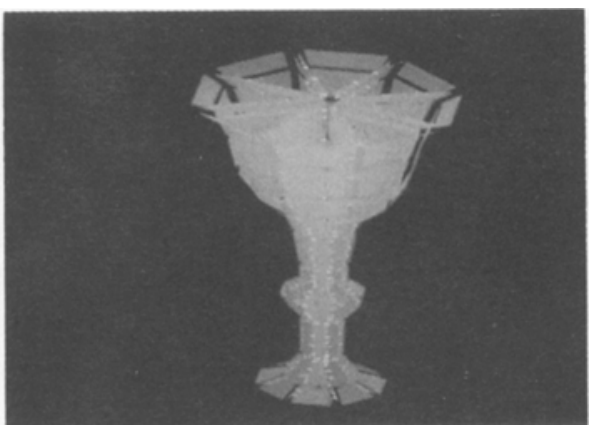

Picture 12: Volumic meshes 